\title{
CONVOLUTION-DOMINATED INTEGRAL OPERATORS
}

\author{
GERO FENDLER \\ Fakultät für Mathematik, Universität Wien \\ Nordbergstrasse 15, A-1090 Wien, Austria \\ E-mail:gero.fendler@univie.ac.at \\ KARLHEINZ GRÖCHENIG \\ Fakultät für Mathematik, Universität Wien \\ Nordbergstrasse 15, A-1090 Wien, Austria \\ E-mail: karlheinz.groechenig@univie.ac.at \\ MICHAEL LEINERT \\ Institut für Angewandte Mathematik, Fakultät für Mathematik \\ Im Neuenheimer Feld 288, D-69120 Heidelberg, Germany \\ E-mail: leinert@math.uni-heidelberg.de
}

\begin{abstract}
For a locally compact group $G$ we consider the algebra $C D(G)$ of convolutiondominated operators on $L^{2}(G)$, where an operator $A: L^{2}(G) \rightarrow L^{2}(G)$ is called convolutiondominated if there exists $a \in L^{1}(G)$ such that for all $f \in L^{2}(G)$

$$
|A f(x)| \leq a \star|f|(x), \quad \text { for almost all } x \in G .
$$

The case of discrete groups was treated in previous publications [6, 7]. For non-discrete groups we investigate a subalgebra of regular convolution-dominated operators generated by product convolution operators, where the products are restricted to those given by multiplication with left uniformly continuous functions. This algebra, $C D_{r e g}(G)$, is canonically isomorphic to a twisted $L^{1}$-algebra.

For amenable $G$ that is rigidly symmetric as a discrete group we show the following result: An element of $C D_{\text {reg }}(G)$ is invertible in $C D_{\text {reg }}(G)$ if and only if it is invertible as a bounded operator on $L^{2}(G)$.

This report is about work in progress. Complete details and further results will be given in a paper still in preparation.
\end{abstract}

2000 Mathematics Subject Classification: Primary 47B35; Secondary 43A20.

Key words and phrases: convolution-dominated operators, inverse-closed subalgebras, symmetry. The paper is in final form and no version of it will be published elsewhere. 
1. Introduction. In these notes we shall consider the following questions:

(1) Is the off-diagonal decay of a matrix inherited by its inverse?

(2) Is the off-diagonal decay of an integral operator inherited by its inverse?

When one considers a convolution operator on the abelian group $\mathbb{Z}$, then its matrix with respect to the natural basis $\left(\delta_{n}\right)_{n \in \mathbb{Z}}\left(\delta_{n}\right.$ denoting the point mass one in $\left.n \in \mathbb{Z}\right)$ is a Toeplitz matrix, i.e., it is constant along the side diagonals. Conversely, a doubly infinite matrix, which is constant along its side diagonals and satisfies certain off-diagonal decay conditions defines a convolution operator, when it is considered as acting with respect to the above basis. For this class of operators and a specific kind of decay (i.e. decay in the sense of $l^{1}$-summability) an answer to the first question is given by the following

THEOREM 1 (Wiener's Lemma). If a two-sided infinite absolutely summable sequence $a=(a(n))_{n \in \mathbb{Z}}$ is invertible as a convolution operator on $l^{2}(\mathbb{Z})$, then the inverse is given by convolution with some $b \in l^{1}(\mathbb{Z})$.

Based on work of de Leeuw [5] and of Bochner and Phillips [3] on operator-valued Fourier transforms, several authors extended Wiener's lemma to the case of matrices for which each side diagonal is uniformly bounded and these bounds are summable. But the index set always had to be a discrete abelian group [1], [18, [19].

In [7] we used tools from abstract harmonic analysis to circumvent the restrictions of abelian Fourier transforms.

THEOREM 2. Let $G$ be a discrete finitely generated group of polynomial growth. If a matrix $A$ indexed by $G$ satisfies the off-diagonal decay condition

$$
|A(x, y)| \leq a\left(x y^{-1}\right), x, y \in G
$$

for some $a \in \ell^{1}(G)$ and $A$ is invertible on $\ell^{2}(G)$, then there exists $b \in \ell^{1}(G)$ such that

$$
\left|A^{-1}(x, y)\right| \leq b\left(x y^{-1}\right), x, y \in G \text {. }
$$

Now we recall the mainline of the proof of this theorem, since this will be a guideline for us.

(i) The matrices satisfying (2) form a Banach $*$-algebra. We denote it by $C D(G)$.

(ii) Identify $C D(G)$ with $l^{1}\left(G, l^{\infty}(G), T\right)$, a twisted $l^{1}$-algebra in the sense of Leptin [12], [13.

(iii) Based on work of Leptin and Poguntke, in particular [15, and on the work of others, we proved that a certain spectral condition for the algebra $C D(G)$ holds true. Namely, that $C D(G)$ is a symmetric Banach *-algebra.

Definition 3. A Banach $*$-algebra $A$ is called symmetric if for all $a \in A$

$$
\sigma_{A}\left(a^{*} a\right) \subset[0, \infty)
$$

where $\sigma_{A}\left(a^{*} a\right)$ denotes the spectrum of $a^{*} a$ in $A$. Accordingly, a locally compact group $G$ is called symmetric, if $L^{1}(G)$ is a symmetric Banach *-algebra. A locally compact (l.c.) group $G$ is called rigidly symmetric if for any $C^{*}$-algebra $A$ the Banach $*$-algebra $L^{1}(G) \hat{\otimes} A$ is symmetric, where the projective tensor product $L^{1}(G) \hat{\otimes} A$ is endowed with its natural $*$-algebra structure. 
(iv) Relate the symmetry of a Banach *-algebra to the invertibility of certain of its elements. This is done with the help of a lemma of Hulanicki [10].

In these notes we generalize Theorem 2 from matrices (indexed by a discrete group) to integral operators on non-discrete locally compact groups. This extension follows the ideas of the discrete case in [7, but requires non-trivial modifications. The special problem to be addressed is the measurability and integrability of certain kernels.

2. Convolution-dominated operators. Let $G$ be a locally compact group endowed with a fixed left Haar measure and the corresponding modular function $\Delta$. For $a \in L^{1}(G)$ we denote by $\lambda(a)$ the operator on $L^{2}(G)$ defined by convolution with $a$ from the left, i.e. $\lambda(a) f=a \star f$, where $(a \star f)(y)=\int_{G} a(x) f\left(x^{-1} y\right) d x, f \in L^{2}(G)$.

Definition 4. A bounded operator $A: L^{2}(G) \rightarrow L^{2}(G)$ is called a convolution-dominated operator if there exists $a \in L^{1}(G)$ such that for all $f \in L^{2}(G)$

$$
|A f(x)| \leq(a \star|f|)(x) \text {, for almost all } x \in G .
$$

We define a norm on the space of convolution-dominated operators by

$$
\|A\|_{C D}=\inf \left\{\|a\|_{1}:(3) \text { holds true }\right\}
$$

and denote by $C D(G)$ the normed linear space of convolution-dominated operators.

Now we collect some properties of convolution-dominated operators.

Proposition 5. The space of convolution-dominated operators $C D(G)$ is a Banach *algebra with the involution of operators on $L^{2}(G)$ and composition of operators as product.

We shall say that a set is locally null if its intersection with any compact set is a null set (see e.g. [9, Definition (11.26)]). Accordingly, a property holds true locally almost everywhere if it holds true except for a locally null set.

Proposition 6. If $A \in C D(G)$ then, there exists a locally integrable function $F_{A}$ : $G \times G \rightarrow \mathbb{C}$ such that for all continuous functions $f$ with compact support we have locally almost everywhere (l.a.e.)

$$
A f(x)=\int_{G} F_{A}(x, y) f(y) d y .
$$

REMARK 1. In terms of the kernel $F_{A}$, the inequality (3) may be rewritten as

$$
F_{A}(x, y) \leq a\left(x y^{-1}\right) \Delta\left(y^{-1}\right), \quad \text { l.a.e. }
$$

Conversely if $F: G \times G \rightarrow \mathbb{C}$ is a locally integrable function, such that for some $a \in L^{1}(G)$

$$
|F(x, y)| \leq a\left(x y^{-1}\right) \Delta\left(y^{-1}\right)
$$

then

$$
A f(x)=\int_{G} F(x, y) f(y) d y
$$

defines a bounded operator on $L^{2}(G)$. This is implied by the Schur conditions:

$$
\text { ess sup } \int|F(x, y)| d y<\infty, \quad \operatorname{ess~sup~}_{y} \int|F(x, y)| d x<\infty .
$$

Clearly, $A$ is dominated in the sense of (3) by convolution with $a$. 
REMARK 2. The kernel of a convolution-dominated operator satisfies the Schur conditions. Therefore it represents the operator, in the sense that for all $f \in L^{2}(G)$ the following integral converges l.a.e. and

$$
A f(x)=\int_{G} F_{A}(x, y) f(y) d y \quad \text { l.a.e. }
$$

REMARK 3. The infimum in (4) is attained.

3. Twisted $L^{1}$-algebra. In this section we shall define a twisted $L^{1}$-algebra and clarify its relation to the convolution-dominated operators.

For a function $f$ on $G$ and $y \in G$ define the left translation $T_{y}$ by

$$
T_{y} f(z)=f\left(y^{-1} z\right), \quad \text { for } z \in G .
$$

Now denote by

$$
\operatorname{Luc}(G):=\left\{f \in L^{\infty}(G):\left\|T_{y} f-f\right\|_{\infty} \rightarrow 0 \text { as } y \rightarrow e\right\}
$$

the algebra of left uniformly continuous functions (note that our notation differs from that of some textbooks e.g. 9]). Then $\operatorname{Luc}(G)$ is a $C^{*}$-algebra with respect to pointwise multiplication on which $G$ acts by left translations. If we endow $\operatorname{Aut}(\operatorname{Luc}(G))$, the group of *-automorphisms of $\operatorname{Luc}(G)$, with the strong operator topology then the map

$$
T: y \mapsto T_{y}, \quad T: G \rightarrow \operatorname{Aut}(\operatorname{Luc}(G))
$$

is continuous. The underlying Banach space of the twisted $L^{1}$-algebra is the projective tensor product

$$
\mathcal{L}=L^{1}(G, \operatorname{Luc}(G), T)=L^{1}(G) \hat{\otimes} \operatorname{Luc}(G) .
$$

The twisted convolution of $h, f \in \mathcal{L}$ is defined by

$$
(h \star f)(x)=\int_{G}\left(T_{y} h(x y)\right) f\left(y^{-1}\right) d y, \quad \text { for } x \in G,
$$

and the twisted involution of $h \in \mathcal{L}$ by

$$
h^{*}(x)=\overline{T_{x^{-1}}\left(h\left(x^{-1}\right)\right)}, \quad \text { for } x \in G .
$$

Next, for $m \in L^{\infty}(G)$ let

$$
D^{m}: L^{2}(G) \rightarrow L^{2}(G), \quad D^{m}(f)=m f,
$$

denote the multiplication operator and set

$$
R: L^{1}(G, \operatorname{Luc}(G), T) \rightarrow B\left(L^{2}(G)\right), \quad R: a \otimes m \mapsto \lambda(a) \circ D^{m} .
$$

A similar calculation as in the proof of [7, Proposition 1] shows the following.

Proposition 7. The map $R: L^{1}(G, \operatorname{Luc}(G), T) \rightarrow B\left(L^{2}(G)\right)$ is an *-algebra homomorphism. It is isometric from $L^{1}(G, \operatorname{Luc}(G), T)$ into $C D(G)$.

Observe that $R$ is not onto $C D(G)$, because we restricted the range algebra to be $\operatorname{Luc}(G)$ and since we use the space of functions Bochner integrable with respect to the norm topology only.

This observation motivates the following definition. 
Definition 8. Elements in the range of $R$ are called regular convolution-dominated operators. We denote the range $R\left(L^{1}(G, \operatorname{Luc}(G), T)\right)$ by $C D_{\text {reg }}(G)$.

The space $C_{0}(G)$ of continuous functions vanishing at infinity is a closed two-sided ideal in $\operatorname{Luc}(G)$, invariant under the operators $\left\{T_{g}, g \in G\right\}$. Hence $L^{1}\left(G, C_{0}(G), T\right)$ is a closed two-sided ideal in $L^{1}(G, \operatorname{Luc}(G), T)$. It follows from [15, Theorem 4] that the Banach *-algebra $L^{1}\left(G, C_{0}(G), T\right)$ is simple and symmetric. The representation $R$ maps $L^{1}\left(G, C_{0}(G), T\right)$ into $\mathcal{K}\left(L^{2}(G)\right)$, the compact operators on $L^{2}(G)$, see [15, Theorem 4]. Moreover

$$
\overline{R\left(L^{1}\left(G, C_{0}(G), T\right)\right)} \|_{o p}=\mathcal{K}\left(L^{2}(G)\right) .
$$

4. Symmetry of the twisted $L^{1}$-algebra. The concept of rigidly symmetric groups was introduced in [15, where it proved useful to show that discrete nilpotent groups are symmetric. Using this concept it is natural to broaden our point of view a little and to admit general $C^{*}$-algebras as the range algebra of the twisted $L^{1}$-algebra.

Let $A$ be a $C^{*}$-algebra and assume that $G$ acts on $A$ continuously by *-automorphisms. Denote $T: G \rightarrow \operatorname{Aut}(A)$ the corresponding homomorphism from $G$ into the *-homomorphisms of $A$. Then we may consider $L^{1}(G, A, T)=L^{1}(G) \hat{\otimes} A$ as a twisted convolution algebra. The formulas for the twisted convolution (6) and the twisted involution (7) remain the same.

THEOREM 9. If $G$ is rigidly symmetric as a discrete group then the twisted algebra $L^{1}(G, A, T)$ is a symmetric Banach *-algebra.

The reader may wonder why the condition on the discretized group $G_{d}$ appears in the hypotheses of the theorem. Following the concept used in [7] one would like to define a map

$$
Q: L^{1}(G, \operatorname{Luc}(G), T) \rightarrow L^{1}(G) \hat{\otimes} B\left(L^{2}(G)\right)
$$

by

$$
Q(f): x \mapsto \lambda(x) \circ D^{f(x)} .
$$

But this does not work since, in general, $x \mapsto \lambda(x) \circ D^{f(x)}$ is not Bochner measurable, because $\lambda: G \rightarrow B\left(L^{2}(G)\right)$ is only strongly continuous but not norm continuous.

The problem can be overcome, completely analogously to [16], by showing that algebraically irreducible representations of a twisted $L^{1}$-algebra of $G$ remain irreducible when "restricted" to the discretized group. Then, as in [16], the characterization of symmetry in terms of the unitarizability of irreducible representations of a Banach $*$-algebra proves the theorem.

If we specialize $T$ to left translation on $\operatorname{Luc}(G)$ then we obtain:

COROLlaRY 10. If $G$ is rigidly symmetric as a discrete group, then $L^{1}(G, \operatorname{Luc}(G), T)$ is a symmetric Banach *-algebra.

5. Invertibility in $C D_{\text {reg. }}$. Finally we study the spectrum of integral operators in $C D_{\text {reg }}(G)$. Let

$$
R: L^{1}(G, \operatorname{Luc}(G), T) \rightarrow C D_{r e g}(G) \subset B\left(L^{2}(G)\right)
$$


be the canonical representation, and let

$$
\lambda^{D}: L^{1}(G, \operatorname{Luc}(G), T) \rightarrow B\left(L^{2}\left(G, L^{2}(G)\right)=B\left(L^{2}(G \times G)\right)\right.
$$

be the $D$-regular representation defined by

$$
\lambda^{D}(f)(\xi)(x, z)=\int_{G}\left(T_{y} f(x y)\right)(z)(\xi)\left(y^{-1}, z\right) d y .
$$

Proposition 11. The representations $\lambda^{D}$ and $R$ of $L^{1}(G, \operatorname{Luc}(G), T)$ are weakly equivalent, i.e.,

$$
\left\|\lambda^{D}(f)\right\|_{B\left(L^{2}\left(G, L^{2}(G)\right)\right)}=\|R(f)\|_{B\left(L^{2}(G)\right.} .
$$

As a representation on $L^{2}(G \times G)$

$$
R^{\omega}(f) \xi(x, z):=\int_{G} f(y)\left(y^{-1} x\right) \xi\left(y^{-1} x, z\right) d y
$$

is just a multiple of $R$. Comparing this to the formula for the $D$-regular representation it is easy to find an intertwining operator between $R^{\omega}$ and $\lambda^{D}$, namely $S \in B\left(L^{2}(G \times G)\right)$ given for $\xi \in L^{2}(G \times G)$ by

$$
S \xi(x, z)=\xi(x z, z) .
$$

COROLlary 12. Let $G$ be an amenable group that is rigidly symmetric as a discrete group. Then for every $f \in L^{1}(G, \operatorname{Luc}(G), T)$ the spectral radius of $f^{*} f$ is given by

$$
r_{L^{1}(G, \operatorname{Luc}(G), T)}\left(f^{*} f\right)=\|f\|_{B\left(L^{2}(G)\right)}^{2} .
$$

We imposed the amenability on $G$ in order to achieve that the largest $C^{*}$-norm on $L^{1}(G, \operatorname{Luc}(G), T)$, denoted $\|$. $\|_{*}$, is just given by the $D$-regular representation. Therefore

$$
\|f\|_{*}=\left\|\lambda^{D}(f)\right\|_{B\left(L^{2}\left(G, L^{2}(G)\right)\right)}=\|f\|_{B\left(L^{2}(G)\right)} \quad \text { for all } f \in L^{1}(G, \operatorname{Luc}(G), T) .
$$

Since $L^{1}(G, \operatorname{Luc}(G), T)$ is symmetric, Pták's theorem [17] asserts that

$$
r_{L^{1}(G, \operatorname{Luc}(G), T)}\left(f^{*} f\right)=\left\|f^{*} f\right\|_{*}=\|f\|_{*}^{2} .
$$

THEOREM 13. Let $G$ be an amenable group that is rigidly symmetric as a discrete group. Then for any operator $A \in C D_{\text {reg }}(G)$

$$
\sigma_{C D}(A)=\sigma_{B\left(L^{2}(G)\right)}(A) .
$$

Since $A=R(f)$ for some $f \in L^{1}(G, \operatorname{Luc}(G), T)$, this follows from the above corollary by an application of Hulanicki's Lemma.

Lemma 1 (Hulanicki). Let $A$ be a Banach $*$-algebra with identity e. Let $T: A \rightarrow B(H)$ be a faithful $*$-representation on a Hilbert space with $T(e)=i d_{H}$. If

$$
\|T(x)\|_{B(H)}=r_{A}(x), \quad \text { for all } x=x^{*} \in A,
$$

then

$$
\sigma_{A}(x)=\sigma_{B(H)}(T(x)), \quad \text { for all } x=x^{*} \in A .
$$

Finally let us recall some examples of amenable groups whose discretization is rigidly symmetric: any abelian group and any nilpotent group is rigidly symmetric as a discrete group. 
Acknowledgments. Most of this work was done while the first named author visited the mathematical Institute of the University of Vienna in April and May 2008.

Gero Fendler and Karlheinz Gröchenig were supported by Marie-Curie Excellence Grant MEXT-CT 2004-517154.

\section{References}

[1] R. Balan, The noncommutative Wiener lemma, linear independence, and spectral properties of the algebra of time-frequency shift operators, Trans. Amer. Math. Soc. 360 (2008), 3921-3941.

[2] A. G. Baskakov, Abstract harmonic analysis and asymptotic estimates for elements of inverse matrices, Mat. Zametki 55 (1992), 17-26.

[3] S. Bochner and R. S. Phillips, Absolutely convergent Fourier expansions for noncommutative normed rings, Ann. of Math. (2) 43 (1942), 409-418.

[4] F. Bonsall and J. Duncan, Complete Normed Algebras, Springer, 1973.

[5] K. de Leeuw, An harmonic analysis for operators. I. Formal properties, Illinois J. Math. 19 (1975), 593-606.

[6] G. Fendler, K. Gröchenig, and M. Leinert, On spectrality of the algebra of convolutiondominated operators, in: Banach Center Publications 78 (2008), 145-149.

[7] G. Fendler, K. Gröchenig, and M. Leinert, Convolution operators on discrete groups, Integr. Equ. Oper. Theory 61 (2008), 493-509.

[8] G. Fendler, K. Gröchenig, M. Leinert, J. Ludwig, and C. Molitor-Braun, Weighted group algebras on groups of polynomial growth, Math. Z. 245 (2003), 791-821.

[9] E. Hewitt and K. A. Ross, Abstract Harmonic Analysis I, Springer Verlag, 1963.

[10] A. Hulanicki, On the spectrum of convolution operators on groups with polynomial growth, Invent. Math. 17 (1972), 135-142.

[11] V. Kurbatov, Functional Differential Operators and Equations, Mathematics and its Applications 473, Kluwer, 1999.

[12] H. Leptin, Verallgemeinerte $L^{1}$-Algebren und projektive Darstellungen lokal kompakter Gruppen I, Invent. Math. 3 (1967), 257-281.

[13] H. Leptin, Verallgemeinerte $L^{1}$-Algebren und projektive Darstellungen lokal kompakter Gruppen II, Invent. Math. 4 (1967), 68-86.

[14] H. Leptin, Darstellungen verallgemeinerter $L^{1}$-Algebren, Invent. Math. 5 (1968), 192-215.

[15] H. Leptin and D. Poguntke, Symmetry and non-symmetry for locally compact groups, J. Funct. Anal. 33 (1979), 119-134.

[16] D. Poguntke, Rigidly symmetric $L^{1}$-group algebras, Seminar Sophus Lie 2 (1992), 189-197.

[17] V. Pták, On the spectral radius in Banach algebras with involution, Bull. London Math. Soc. 2 (1970), 327-334.

[18] J. Sjöstrand, Wiener type algebras of pseudodifferential operators, in: Séminaire sur les Équations aux Dérivées Partielles, 1994-1995, Exp. No. IV, École Polytech., Palaiseau, 1995.

[19] Q. Sun, Wiener's lemma for infinite matrices, Trans. Amer. Math. Soc. 359 (2007), 30993123 . 
